\title{
Flexible head, designed for measuring residual stress along variable curves using ultra-sonic transducers
}

\section{DANIEL Laurent ${ }^{1, a^{*}}$ and BELAHCENE Farid ${ }^{2, b}$}

${ }^{1}$ Institut Charles Delaunay, Laboratoire des Systèmes Mécaniques et d'Ingénierie Simultanée, Université de Technologie de Troyes, 12 rue Marie Curie, 10000 TROYES, France.

2ULTRA-RS, 12, rue Gustave Eiffel, 10430 ROSIERES PRES TROYES, France.

alaurent.daniel@utt.fr*, bf.belahcene@ultrars.com

Keywords: ultra-sounds, residual stress measurement, curve following device.

Abstract. Ultra-sonic measure of residual stress has been along for a while. One main drawback of this technique is the need for a measuring head adapted to each new surface shape. We introduce a new measurement head designed to adapt to changing curves. This device will open the possibility of curved surfaces residual stress cartography. We focus on the device geometric design, its possibilities and limitations.

\section{A device to measure stress along variable curves.}

Fig. 1 shows a classical ultra-sonic measuring chain that could allow stress measurement on variable curves. It is the same as those used with flat parts or regular cylinders, like detailed in [1] to [5]. The difference is a special head that will adapt to variable curves shown in Fig. 2.
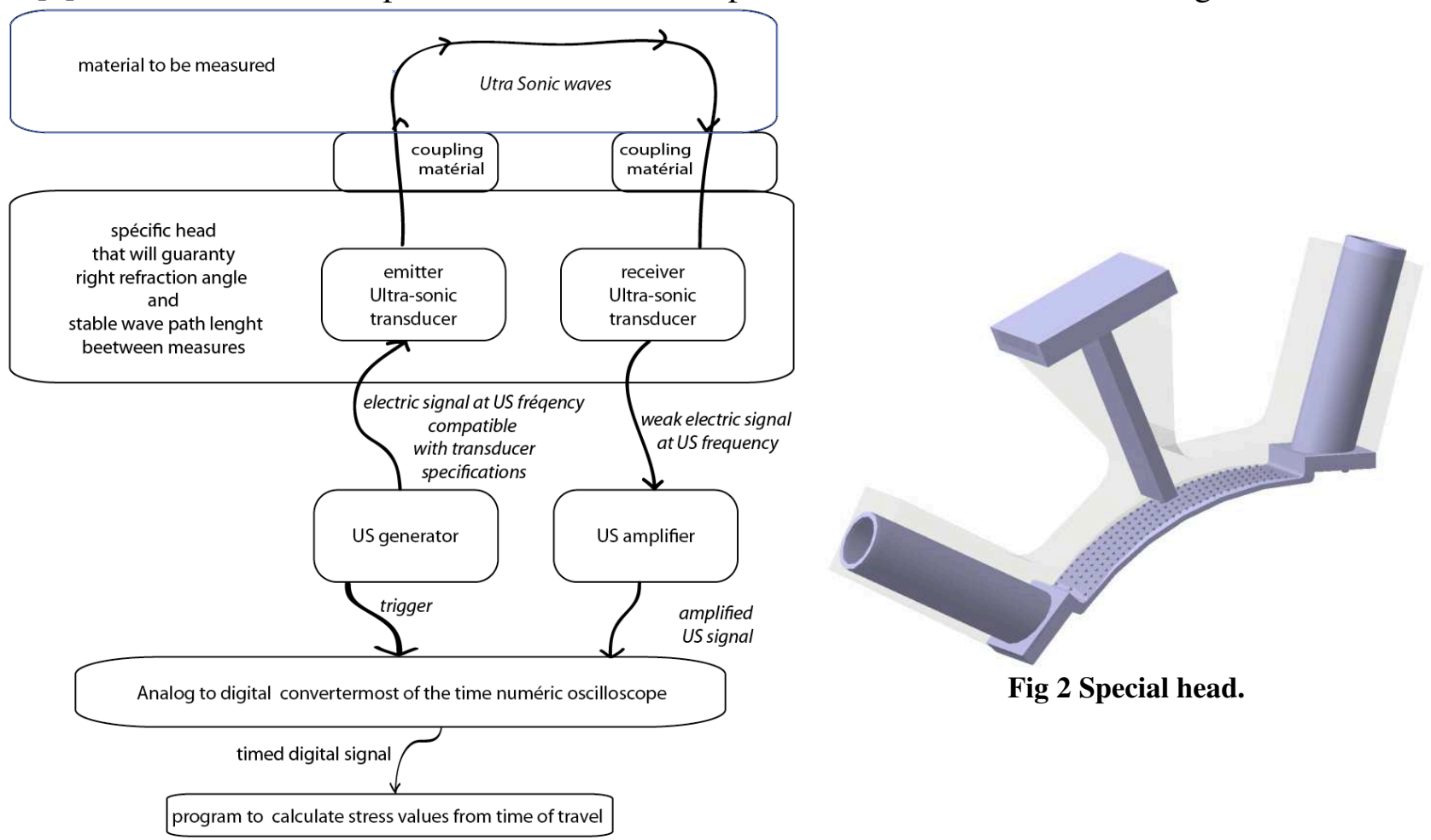

Fig 2 Special head.

Fig 1 Ultra-sonic measuring chain

\section{The changing curve problem}

Devices used to hold piezo transducers while measuring residual stress with ultra-sonic method shall ensure that wave path length both into coupling fluid and along measured part surface, between the emitting and receiving transducers, keep the same between measures. That will guaranty that the sole variable parameter will be the time of flight of ultra-sonic signal into the 
studied material induced by stress variation between measurement points. Solid transducer holders can guaranty that while measuring along flat or regularly rounded surfaces. But so far, no device existed that was able to keep theses wave path length correctly, while measuring along variable curves. The absolutely ideal device would guaranty that the three parts of the wave path, from transceiver to receptor into water, then metal, then water, keep exactly the same between two measures. To solve that, it should fulfill three main requirements for each measurement point:

- Guarantying that wave path length (D in Fig. 3) in between transducer and metal surface into water remain the same.

- Guarantying that surface wave path length along material surface ( $C L_{p}$ in Fig. 3$)$ remains the same.

- Guarantying that the local surface to transducer incidence angle ( $\alpha$ in Fig. 3 ) conforms to expected refraction angle between coupling material and measured material.

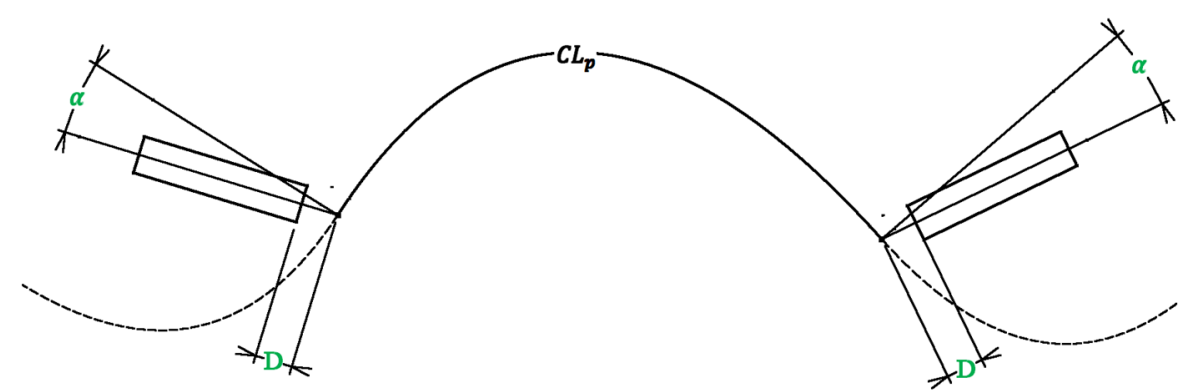

Fig. 3 Geometric constraints to fulfill (transducers shown as rectangles).

\section{Device to adapt to curve variation}

The device shown on Fig. 2, designed to fulfill these three requirements is made as follows:

- Two transducers holders made of a tube extended by a contact system that guaranty stable distance and contact angle between transducer surface and material surface along curves.

- A thin strip that has to be kept in contact with the material surface along the path of the wave. It guaranty stable surface distance between transducers whatever is the surface curve.

\section{System limitations}

Limitations are mainly due to geometric considerations of two kinds. One has to do with the local point of contact distance variation in between transducer and metal surface due to the very local variation of surface curve as illustrated in Fig. 4 and 5. The other is linked to the difference in between the actual length of the strip that maintains distance between transducer and the real path of the wave. Here again that difference vary with the curve as illustrated in Fig. 6.

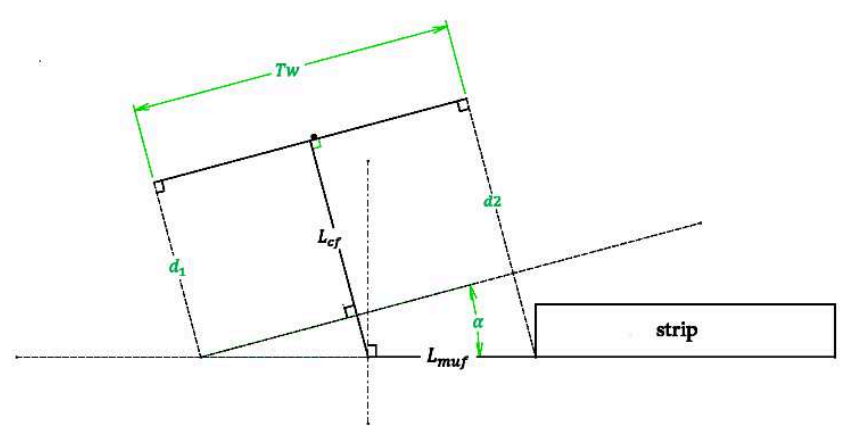

Fig. 4 Flat part case.

In Fig. 4, flat part case, correct angle $\alpha$ is maintained while pressing the system along part surface in order to maintain a set of two touch-points in contact with the measured part surface. Respective distances from transducer to points of contact are named $d_{1}$ and $d_{2}$ and inter-touch point width at transducer level named $T w$. The difference between $d_{1}$ and $d_{2}$, used to maintain correct refraction angle, $\alpha$ from transducer surface to part surface is calculated as follows:

$$
d_{2}=d_{1}+T w \cdot \tan \alpha
$$


Wave path length from strip border to transducer center is equal to part going from transducer to material surface plus the part going from material surface wave contact point to strip border. Since both coupling fluid and measured part are different materials, they present different wave speed propagation properties, characterized by their accousto-elastic constants. Therefore we calculate wave path length into coupling fluid, $L_{c f}$ and wave path length in material under fluid, $L_{m u f}$. Their respective equations for flat case are:

$$
\begin{aligned}
& L_{m u f}=\frac{T w}{2 \cos \alpha} . \\
& L_{c f}=\frac{d 1+d 2}{2} . \quad L_{c f}=d_{1}+L_{m u f} \cdot \sin \alpha .
\end{aligned}
$$

Fig. 5 shows wave path variation induced by the curve variation at local point of contact for a concave curve. The radius is voluntarily set to an unrealistic value being quite similar to $T w$. This is to enlarge enough the space between lines. Actual wave path is shown in red. We split this path into a new wave path length in coupling fluid, $N L_{c f}$ and new wave path length into material under fluid, $N L_{m u f}$. $N L_{c f}$ is linked to the Fig. 5 indigo triangle side length named $c$ by the following formula:

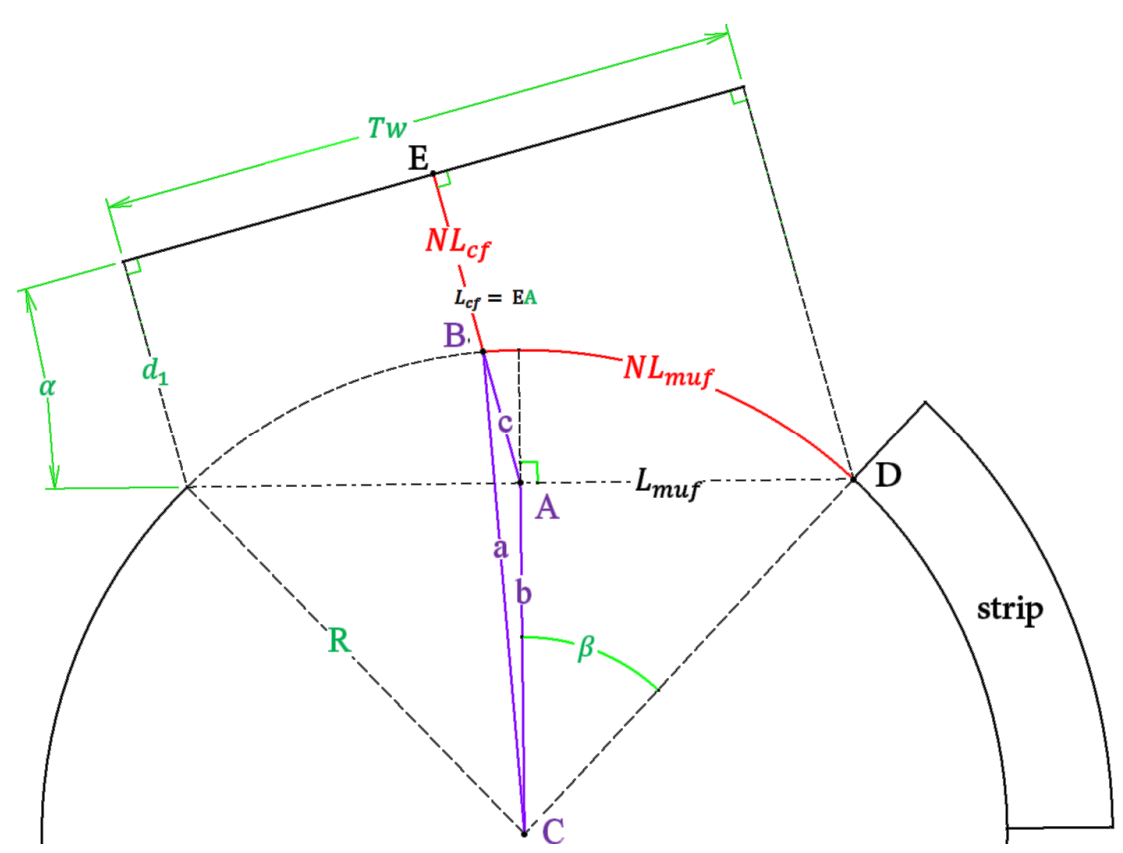

Fig. 5 Local point of contact curve variation problem, concave case.

$$
N L_{c f}=L_{c f}-c
$$

$N L_{m u f}$ is equal to the length of circle portion determined by $\beta$ angle plus length of circle portion determined by indigo triangle $\hat{C}$ angle, that summarized into following formula with $R$ being the local radius and angles expressed in radians:

$$
N L_{m u f}=(\beta+\hat{\mathrm{C}}) \cdot R \text {. }
$$

In order to find $N L_{c f}$ and $N L_{m u f}$ we shall find, $\beta$ and $\hat{C}$ values. $\beta$ is given by the following relation between local radius $\mathrm{R}$ and previously established $L_{m u f}$ :

$$
\beta=\sin ^{-1}\left(L_{m u f} / R\right)
$$

Both $c$ and $\hat{\mathrm{C}}$ values are established using relations in indigo triangle provided that values of $\hat{\mathrm{A}}$ angle, and length $\mathrm{a}$ and $\mathrm{b}$ of that indigo triangles are known. $\hat{\mathrm{A}}$ comes from $\alpha$ following the formula:

$$
\hat{\mathrm{A}}=\pi-\alpha .
$$


Value of $a$ is equal to $R$, and $b$ is found by:

$$
b=\cos \beta \cdot R \text {. }
$$

$\hat{A}$ angle, and length $a$ and $b$ being given, we can now use "side to sinus angle ratios" in Fig. 5 indigo triangle:

$$
\frac{a}{\sin \hat{A}}=\frac{b}{\sin \hat{B}}=\frac{c}{\sin \hat{C}} \quad, \quad \sin \hat{B}=b \cdot \frac{\sin \hat{A}}{a} \quad, \hat{B}=\sin ^{-1}\left(b \cdot \frac{\sin \hat{A}}{a}\right) .
$$

Using sum of angles into triangles $\hat{C}$ will be:

$$
\hat{\mathrm{C}}=\pi-(\hat{\mathrm{A}}+\hat{B}) \text {. }
$$

And now that we got $\hat{C}$ we can use again "side to sinus angle ratios" for $c$ :

$$
c=a \cdot \frac{\sin \hat{\mathrm{C}}}{\sin \hat{\mathrm{A}}} \text {. }
$$

The direct wave path lengths formulas for $N L_{c f}$ and $N L_{m u f}$ with $T w, d_{1}, \alpha$ and $R$ as entry points will be deduced by successive replacements of previous formulas :

$$
N L_{c f}=L_{c f}-c=d_{1}+\frac{T w}{2 \cos \alpha} \cdot \sin \alpha-R \cdot \frac{\sin \left(\alpha-\sin ^{-1}\left(\sqrt{1-\left(\frac{T w}{2 R \cos \alpha}\right)^{2}} \cdot \sin \alpha\right)\right)}{\sin \alpha}
$$

$N L_{c f}$ being established we still have to find $N L_{m u f}$. It is function of angle $\hat{C} \& \beta$ and radius $R$ through the following angle-to-arc-length relation :

$$
\begin{aligned}
& N L_{\text {muf }}=(\beta+\hat{\mathrm{C}}) \cdot R \\
& N L_{m u f}=\left(\sin ^{-1}\left(\frac{T w}{2 R \cos \alpha}\right)+\alpha-\sin ^{-1}\left(\sqrt{1-\left(\frac{T w}{2 R \cos \alpha}\right)^{2}} \cdot \sin \alpha\right)\right) \cdot R .
\end{aligned}
$$

\section{Curve effect on variation of strip length by comparison to real wave path length.}

The specially designed head use a thin strip. That strip is designed to be thin enough in order to be closely maintained against the surface of part to be measured. That way the strip length will reflect the length of wave path next from surface under it. Since the strip has a certain thickness named $h$ on Fig. 6 , while bent, the side positively bent extends and the other one retract. Strip neutral fiber length, $L n$ keeps the same. The actual length of the part that is next from the surface $L s$ will vary according to radius $R$ of local curve under the strip like follows with sign being minus for bumps and plus for holes:

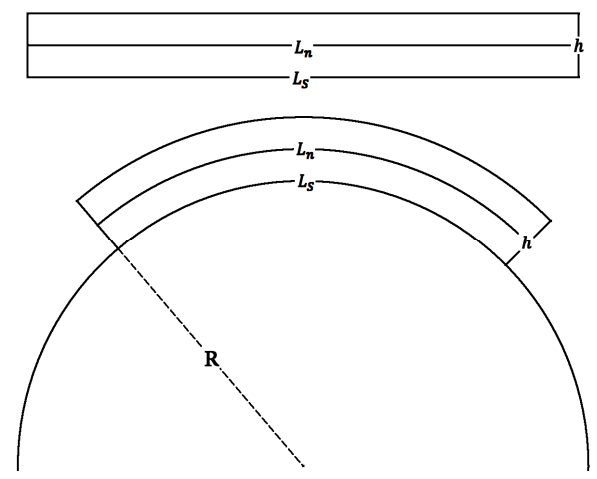

Fig. 6 Curve effect on variation of strip to real path distance ratio.

$$
L_{S}=L_{n} \pm \frac{L_{n} h}{2 R+h}
$$




\section{Total corrected wave path lengths in coupling fluid and material}

By the end two values are calculated. $C L_{c f}$ is the total corrected wave path length into coupling fluid, and $C L_{p}$ is the total corrected wave path length into measured part. $C L_{c f}$ is the sum of wave path length in coupling fluid for each transceiver, at each side of the device $N L_{c f(1)}$ and $N L_{c f(2)}$. Its formula is:

$$
C L_{c f}=N L_{c f(1)}+N L_{c f(2)}
$$

$C L_{p}$ is the sum of wave path length into material under fluid for each transceiver, $N L_{m u f(1)}$ and $N L_{m u f(2)}$, at each side of the device, plus the actual length of the part that is next from the surface of the strip $L_{s}$.

$$
C L_{p}=N L_{m u f(1)}+N L_{m u f(2)}+L_{s} .
$$

When calculating time of travel, these values will be used with measured part material and coupling fluid accousto-elastic constants in order to correct total time of flight value.

\section{Ultra-sonic wave refraction angle variation induced by local radius variation.}

Fig. 7 is issued from Fig. 6 and shows how part local radius influences wave incidence at interface in between coupling fluid and part surface.

New incidence angle, $\alpha_{n}$ will be function of original one, $\alpha$ and radius, $R$.

$$
\begin{aligned}
& \alpha_{n}=\hat{B} \\
& \alpha_{n}=\sin ^{-1}\left(\sqrt{1-\left(\frac{T w}{2 R \cos \alpha}\right)^{2}} \cdot \sin \alpha\right) .
\end{aligned}
$$

That angle variation will mainly have an impact on quantity of energy transmitted from emission to reception transducers.

\section{Thermal considerations}

Temperature has an impact on measure accuracy. Depending on strip material dilatation coefficient, an error can be generated. If strip material dilatation coefficient is similar to measured material one, this error is kept to a minimum since both materials behave the same. Otherwise error coefficient, $\varepsilon$, is proportional to ratio between the strip material and measured part material ones, $\alpha_{s}$ and $\alpha_{p}$ :

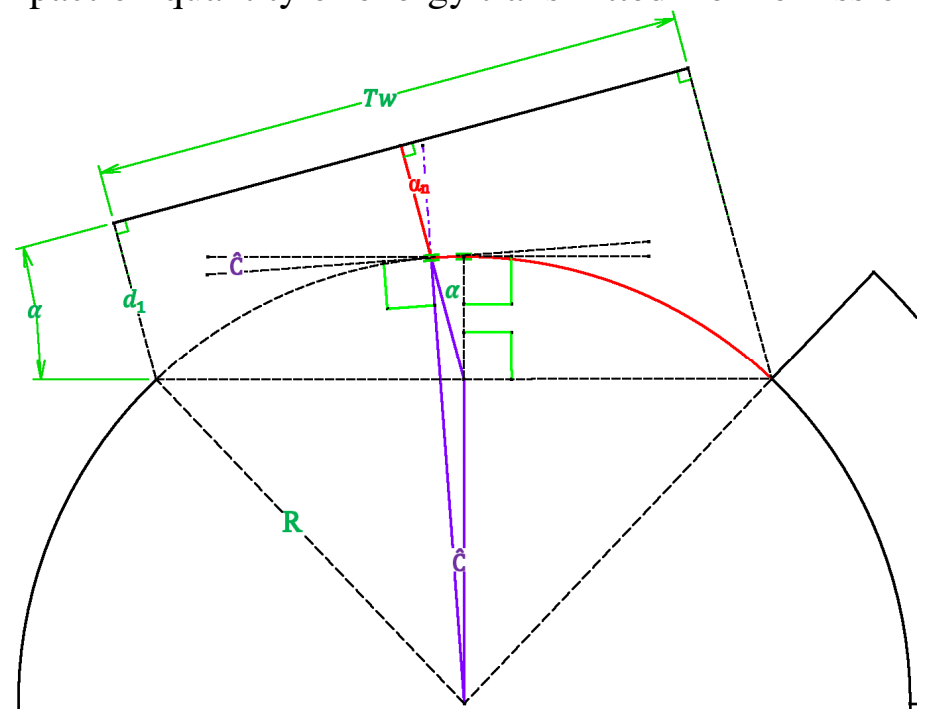

Fig. 7 Radius influence on wave incidence at fluid to part interface.

$$
\varepsilon=\frac{\alpha_{s}}{\alpha_{p}} .
$$


That known, for measures to be as accurate as possible, both part and device temperature should be kept the same along measuring campaign. Difference in thermal dilatation coefficient between measuring head and part has then no impact on relative results between measurements.

\section{Conclusion: future steps toward validation}

Our first prototype was too rigid. We will build a new one, flexible enough to allow our proposed geometric correction factors to be realistic. That one build we will have to validate our calculus by using it upon parts presenting known stress values. Calculus show that with wave speed travel of $5930 \mathrm{~m} . \mathrm{s}^{-1}$ in steel and a measuring range of $30 \mathrm{~m} \cdot \mathrm{s}^{-1}$ being equivalent to $420 \mathrm{MPa}$ range, for intertransducers distance of $100 \mathrm{~mm}$, a distance variation of $0.5 \mathrm{~mm}$ would cover the whole range. Hence to achieve a 10 points resolution along our range we should know inter-transducer distance with an accuracy of $0.05 \mathrm{~mm}$. This seems achievable at first sight by using proposed geometric correction method.

\section{Perspectives: getting to a complete residual stress cartographic device}

We saw that main system drawbacks are errors induced by geometrical variations of wave path length both into coupling fluid and into material due to surface curve. Since ultra-sonic measuring system rely on wave time of travel variations according to stress variations, wave path length should be kept the same in between measures. However we saw that this is not achievable. Our system could only get usable if used with solution for aggregating data points and establish a map of local curves precise enough to correct time of flight measures using the relations we established between known device geometric informations and their impact on device size variation. If changes in wave time of flight due to corrected wave path length prove to be accurate enough for being negligible against changes in wave time of flight dues to stress variation, then we would achieve correct measurement.

Even that provided, aggregation of stress data is more complex that mapping found stress values to points of measure since the raw values that are given back by the system do not reference points but strips along material surface. A new treatment of strip position and orientation and stress value given for each position has to be conducted in order to get back to actual stress map of material surface. This system will require our special measuring head to be adapted to a commercial 3D measurement device in order to aggregate the following parameters XYZ position of head, strip orientation along surface, and average stress value along strip. Such a system has been patented [6] by authors.

\section{References}

[1] R. B. Thompson, J. Lu. W.Y. and Clark. A.V., ultrasonic methods, handbook of measurement of residual stresses, ed. by J. Lu, SEM, inc.,149-178, 1996.

[2] F. Belahcene, Détermination des contraintes résiduelles superficielles par méthode ultrasonore, Thèse de doctorat, UTC, Compiègne, France, 2000.

[3] Xiaolai Zhou, “ Etude paramétrique pour la détermination des contraintes résiduelles par la méthode ultrasonore ", Thèse de doctorat, UTT, Troyes, Françe, 2006.

[4] F. Belahcene, J. Lu, Determination of residual stress using critically refracted longitudinal waves and immersion mode, journal of strain analysis for engineering design, 37 (1), 13-20, 2002.

[5] F. Belahcene, F. Thomas, J. Lu, X. Zhou, ”Determination of compressive residual stresses using critically refracted longitudinal (LCR) waves", American Society of Mechanical Engineers, Pressure Vessels and Piping Division (Publication) PVP, 484, 137-140, 2004.

[6] L. Daniel, F. Belhacene, Irregular parts subsurface residual stress measuring set, Patent FR1354617 deposit date 22/05/2013. 